\title{
Frustration-mediated superconductivity in two-dimensional classical Heisenberg model
}

\author{
K Y SZETO \\ Department of Physics, York University, Toronto, Ontario, Canada M3J 1P7
}

\begin{abstract}
The two-dimensional frustrated classical Heisenberg model on a square lattice is used to describe the magnetic interaction in $\mathrm{La}_{2} \mathrm{CuO}_{4}$. In the slow-hopping regime, the model suggests an $X Y$-like ground state. An analysis of the effective interaction between frustrated bonds shows the possibility of pair formation and superconductivity.
\end{abstract}

Keywords. Magnetic pairing; Heisenberg model; frustration.

One common approach to understand high temperature superconductivity (Emery 1989) of $\mathrm{La}_{2} \mathrm{CuO}_{4}$ begins by assuming a Hamiltonian with two terms: a kinetic term $E_{k}$ describing the hopping of the holes and an antiferromagnetic exchange interaction $J$. Since $\mathrm{La}_{2} \mathrm{CuO}_{4}$ has a layered structure that can be approximated as coupled $\mathrm{Cu}$ planes with strong intraplanar and weak interplanar interaction, we can use a two-dimensional model for the main magnetic interaction. Assuming a large effective mass for the holes (Blezius and Carbotte 1987; Schossmann et al 1987; Ramirez et al 1987), we consider the limit of slow hopping. We use a quasi-static picture of the hole sitting on the oxygen site at the midpoint of the $\mathrm{Cu}-\mathrm{Cu}$ bond and hops to the nearest neighbouring oxygen sites (Aharony et al 1988). A simple Hamiltonian for this scenario is an antiferromagnetic Heisenberg model defined on a square lattice with dilute frustrated bonds at $\left\{\left(\mathbf{r}_{i}, \mathbf{r}_{j}\right)\right\}$ created by holes located at their midpoints,

$$
H=-J \sum_{\left\langle\mathbf{r}^{\prime}\right\rangle} \mathbf{s}(\mathbf{r}) \cdot \mathbf{s}\left(\mathbf{r}^{\prime}\right)+(K+J) \sum_{\left\langle\mathbf{r}_{,}, \mathbf{r}_{j}\right\rangle} \mathbf{s}\left(\mathbf{r}_{i}\right) \cdot \mathbf{s}\left(\mathbf{r}_{j}\right)
$$

Here $\mathbf{r}=m_{x} \hat{x}+m_{y} \hat{y}$ defines discrete point on a square lattice on which sits a unit magnitude classical three-component spin $\mathbf{s}$. The first sum is over all the nearest neighbours and the second sum is over only the set of neighbours of the sites $\left\{\left(\mathbf{r}_{i} \mathbf{r}_{j}\right)\right\}$. The effective $K$ is the ferromagnetic exchange between the frustrated $\mathrm{Cu}$ spins at $\left(\mathbf{r}_{i}, \mathbf{r}_{j}\right)$. We only consider the special case for $K \gg J$, which is most relevant to $\mathrm{La}_{2} \mathrm{CuO}_{4}$ (Aharony et al 1988; Birgeneau et al 1988). In the slow-hopping limit, the average time between hop is proportional to $1 / E_{K}$ which is much larger than the time $1 / J$ for the $\mathrm{Cu}$ spins to flip and adjust to the new environment created by the holes. Consequently, when these holes hop, the frustrated bonds follow. The size of the frustrated area around the hole depends on the relative magnitude of $E_{K}$ and $J$ and its calculation requires a detailed microscopic theory.

First we consider the ground state of $H$. In general, we can represent $\mathbf{s}(\mathbf{r})$ by spherical coordinates $(\phi(\mathbf{r}), \theta(\mathbf{r}))$ so that

$$
\mathbf{s}\left(\mathbf{r}_{1}\right) \cdot \mathbf{s}\left(\mathbf{r}_{2}\right)=\cos \left(\phi\left(\mathbf{r}_{1}\right)-\phi\left(\mathbf{r}_{2}\right)\right) \sin \left(\theta\left(\mathbf{r}_{1}\right)\right) \sin \left(\theta\left(\mathbf{r}_{2}\right)\right)+\cos \left(\theta\left(\mathbf{r}_{1}\right)\right) \cos \left(\theta\left(\mathbf{r}_{2}\right)\right) \text {. }
$$

Note that $0 \leqslant \sin \left(\theta\left(\mathbf{r}_{1}\right)\right) \sin \left(\theta\left(\mathbf{r}_{2}\right)\right)$ because $0 \leqslant \theta \leqslant \pi$. The minimum of $H$ is achieved when the prefactor $\cos \left(\phi\left(\mathbf{r}_{1}\right)-\phi\left(\mathbf{r}_{2}\right)\right)$ in (2) is 1 . This implies that

$$
\mathbf{s}\left(\mathbf{r}_{1}\right) \cdot \mathbf{s}\left(\mathbf{r}_{2}\right)=\cos \left(\theta\left(\mathbf{r}_{1}\right)-\theta\left(\mathbf{r}_{2}\right)\right)
$$


is a necessary condition for the minimum energy spin configuration. Thus the ground-state is $X Y$-like. It is not Ising-like as in the unfrustrated case because in general the spins on the frustrated bond defined a different direction $\hat{k}$ from the direction of the other unfrustrated spins. Since the ground state is $X Y$-like, the spin configuration can be described by a single spin angle $\psi$. For the special case of only one frustrated bond, the analytical expression (Szeto and Wu 1990) for the spin angle in the continuous limit of the lattice is

$$
\psi(x, y)=\frac{\pi}{2} \frac{\varepsilon x}{x^{2}+y^{2}}
$$

where $\varepsilon$ is the radius of the core of frustration containing a frustrated bond along $\hat{x}$. For the case with $n$ static frustrated bonds of strength $K \gg J$, the $X Y$ nature of the ground state can be established in a similar manner. We can numerically obtain the spin configuration by means of a cellular automata algorithm consisting of the following rules for each spin,
(a) $\mathbf{s}(|\mathbf{r}| \rightarrow \infty)= \pm \hat{\imath}$
(b) $\mathbf{s}\left(\mathbf{r}_{i}\right) \| \mathbf{s}\left(\mathbf{r}_{j}\right)$
(c) $\mathbf{s}(\mathbf{r})=-\hat{B}(\mathbf{r})$.

Here rule (a) means that the spins far away from the frustrated regions (taken to be near the origin) are antiparallel, as it should for the ground state of an unfrustrated Heisenberg antiferromagnet. Rule (b) implies that on the frustrated bond $\left(\mathbf{r}_{i}, \mathbf{r}_{j}\right)$, the strong ferromagnetic coupling $(K \gg J)$ enforces the pair of spins at the endpoints of this bond to behave collectively as a unit. Rule (c) requires the definition of the average magnetic field B. First, for spins not sitting at the endpoints of the frustrated bonds, $\mathbf{B}$ is the vector sum of the four nearest neighbours of $\mathbf{s}$. When the spin is sitting on a frustrated bond, $B$ is the sum of the six nearest neighbours of the two frustrated spins on the endpoints of the frustrated bond. When $\mathbf{B}=\mathbf{0}$, the orientation of $\mathbf{s}$ is unchanged. This set of rules for the evolution of the spin configuration will reach a local energy minimum state, as any small perturbation from the evolved state raises the energy. Since the ground state of an unfrustrated classical antiferromagnet is the Néel state, the antiferromagnet with frustration should also be determined by global spin arrangement similar to the Néel state so that the boundary condition (rule (b)) around the core of the frustrated region is satisfied. The local energy minimum rules (a) and (c) provide the necessary readjustment of the spins orientation from the Néel state. Based on this reasoning, we believe that this local energy minimum state is actually the ground state. The numerical calculation on a $20 \times 20$ lattice for various distribution of frustrations is performed and we conclude that the ground state is indeed $X Y$-like. In the continuous limit, we have defined a homotopy group for the case of a ferromagnet with antiferromagnetic frustrated bonds (Szeto and Wu 1990). Since the square lattice of $\mathrm{La}_{2} \mathrm{CuO}_{4}$ can be divided into sublattices, we expect that a similar definition for antiferromagnet with ferromagnetic frustration can be made. Once the homotopy group is defined, the analysis of topological invariants such as instantons can be made and related to local energy minimum states, although the neat results of Belavin and Polyakov (1975) cannot carry through because of the 
complicated boundary conditions around the core of frustrations that the global spin configurations must satisfy.

These theoretical observations imply that at least at sufficiently low temperature, the Heisenberg nature of the magnetic exchange in (1) is drastically changed to an $X Y$ exchange as soon as frustration is introduced. Experimentally, the $X Y$ nature of the low temperature magnetic state of $\mathrm{La}_{2} \mathrm{CuO}_{4}$ is evident in the analysis of the susceptibility data using high temperature series (Szeto 1990). The goodness-of-fit for the zero-field susceptibility data of $\mathrm{La}_{2} \mathrm{CuO}_{4}$ (Mitsuda et al 1987; Johnston et al 1987; Endoh et al 1988) indicates that the $X Y$ model is a good starting Hamiltonian to describe the magnetic interaction between the $\mathrm{Cu}$ spins. This is also in agreement with the observed $\mathrm{Cu}$ spin arrangement by Vaknin et al (1987).

After the above justification for the $X Y$ nature of the ground state of the two-dimensional frustrated Heisenberg antiferromagnet, we can begin the analysis of $\mathrm{La}_{2} \mathrm{CuO}_{4}$ with the $X Y$ model. The advantage of this approach is that many techniques on the $X Y$ model can be used for the analysis of frustrated plaquettes, which we show to exhibit attractive pairing interaction between frustrated bonds, i.e. the holes in the original context of $\mathrm{La}_{2} \mathrm{CuO}_{4}$ (Szeto 1989). This attractive interaction is also confirmed by the cellular automata simulation, where the minimum energy spin configuration with two frustrated bonds at various separation and orientations are calculated. In the $X Y$ model, the frustrated plaquettes can be assigned chiralities and the interaction between chiralities is logarithmic. By a summation of these logarithmic interaction between the two frustrated plaquettes on two sides of the frustrated bonds we can obtain the effective interaction between frustrated bonds. The lowest order of this effective interaction is dipolar, as expected from the analogy of the two frustrated plaquettes as two charges forming a dipole that runs across the frustrated bond. A BCS mean-field calculation with the dipolar interaction with experimental input parameters yields a phase diagram for critical temperature versus hole density that compares favourably with experiments (Szeto 1989).

These numerical and theoretical investigations into the ground-state properties of Heisenberg magnets with frustrations indicate the relevance of the $X Y$ model in the study of the classical spin systems. These results should have relevance in the corresponding quantum systems as we replace the classical values of $J$ and $K$ by the renormalized ones (Chakravarty et al 1988).

\section{Acknowledgements}

Discussion with Prof. Y Wu is appreciated. KYS acknowledges support by the National Science and Engineering Research Council of Canada through a University Research Fellow grant.

\section{References}


Emery V J 1989 Mater. Res. Soc. Bull. XIV 67

Endoh Y et al 1988 Phys. Rev. B37 7443

Johnston D C et al 1987 Phys. Rev. $\mathbf{B 3 6} 4007$

Mitsuda S et al 1987 Phys. Rev. B36 822

Schossmann M et al 1987 Phys. Rev. B36 3627

Ramirez A P et al 1987 Phys. Rev. B35 8833

Szeto K Y 1989 Physica C161 527

Szeto K Y 1990 Mod. Phys. Lett. 84283

Szeto K Y and Wu Y 1990 J. Phys.; Condens. Matter 21037

Vaknin D et al 1987 Phys. Rev. Lett. 582802 